\title{
Pricing of barrier options by marginal functional quantization
}

\author{
Abass SAGNA* \\ Laboratoire d'Analyse et de probabilités de \\ l'Université d'Evry Val d'Essonne \& \\ ENSIIE
}

\begin{abstract}
This paper is devoted to the pricing of Barrier options by optimal quadratic quantization method. From a known useful representation of the premium of barrier options one deduces an algorithm similar to one used to estimate nonlinear filter using quadratic optimal functional quantization. Some numerical tests are fulfilled in the Black-Scholes model and in a local volatility model and a comparison to the so called Brownian Bridge method is also done.
\end{abstract}

\section{Introduction}

Consider a fixed time horizon $T$, which will be typically the maturity of the option in a financial model, and let $(\Omega, \mathcal{F}, \mathbb{P})$ be a probability space (modeling the randomness of the market) with a filtration $\mathcal{F}=\left\{\mathcal{F}_{t}, 1 \leq t \leq T\right\}$ satisfying the usual requirements. The probability $\mathbb{P}$ is supposed to be the probability in the 'real world' in opposite to the risk neutral probability.

Consider that the stock price process $\left(X_{t}\right)_{t \in[0 . T]}$ satisfies the following time homogenous stochastic differential equation (SDE)

$$
d X_{t}=b\left(X_{t}\right) d t+\sigma\left(X_{t}\right) d W_{t}, \quad X_{0}=x \in \mathbb{R},
$$

where $\left(W_{t}\right)_{t \in[0, T]}$ denotes a one-dimensional Brownian motion defined on the probability space $(\Omega, \mathcal{F}, \mathbb{P}) ; b: \mathbb{R} \rightarrow \mathbb{R}$ and $\sigma: \mathbb{R} \rightarrow \mathbb{R}$ are continuous functions satisfying the global Lipschitz and linear growth conditions:

$$
|b(x)-b(y)|+|\sigma(x)-\sigma(y)| \leq C|x-y|
$$

and

$$
|b(x)|+|\sigma(x)| \leq C(1+|x|),
$$

for every $t \in[0, T]$ and for every $x, y \in \mathbb{R}$. The filtration considered here is the natural filtration of the brownian motion completed by the $\mathbb{P}$-null sets.

It is known that under the above assumptions on the coefficients of the diffusion there exists a unique strong solution for the SDE (see e.g. [12, 16]). The uniqueness of the solution is ensured by the global Lipschitz assumption (1.2) whereas the linear growth assumption (1.3) guaranties that this solution do not explode (see [16] for more details).

*E-mail: abass.sagna@gmail.com. This research is supported by the "Chaire Risque de Crédit" of the French Banking Federation. 
The first workable model for 'rational' market pricing of traded options have been proposed by Black-Scholes in 1973 and extended by Merton in the same year. In the Black-Scholes model the economics consists on two assets: the stock price with dynamics as the previous SDE with $b(t, x):=$ $\mu x$ and $\sigma(t, x):=\sigma x$, and a zero-coupon bound of constant interest rate $r$ and maturity $T$.

Moreover, we know that under arbitrage free and completeness assumptions, the discounted price at time $t$ of any European contingent claim is uniquely determined and is the expectation, under a probability $\widetilde{\mathbb{P}}$ called risk neutral probability, of its discounted payoff (a functional of the price process $\left(X_{t}\right)_{t \in[0, T]}$ which may depend on all the trajectory of the process), given all the information available up to time $t$. If $V_{t}$ is the value of the option a time $t$ and if $h$ denotes the payoff at the maturity, then

$$
V_{t}=e^{-r(T-t)} \mathbb{E}\left(h \mid \mathcal{F}_{t}\right)
$$

where $\mathbb{E}$ is the expectation under $\widetilde{\mathbb{P}}$, so that the price at time 0 is

$$
V_{0}=e^{-r T} \mathbb{E}(h)
$$

Our aim in this work is to estimate such an expectation for a class of path-dependent payoffs: barrier options, by optimal quantization method. We consider here a class of exotic options whose payoff depend on both the value of the underlying asset at the maturity and its maximum or its minimum over $[0, T]$. This means, payoffs $h$ of the form

$$
h=F\left(X_{T}, \sup _{t \in[0, T]} X_{t}\right) \quad \text { or } \quad h=F\left(X_{T}, \inf _{t \in[0, T]} X_{t}\right) .
$$

When the payoff can be decomposed as

$$
h=\varphi\left(X_{T}\right) \mathbf{1}_{\left\{\sup _{t \in[0 . T]} X_{t} \in I\right\}} \quad \text { or } \varphi\left(X_{T}\right) \mathbf{1}_{\left\{\inf _{t \in[0 . T]} X_{t} \in I\right\}}
$$

where $I$ is an unbounded interval of $\mathbb{R}$, one speaks about barrier options. This last class is a particular case of payoffs of the form

$$
h=\varphi\left(X_{T}\right) \mathbf{1}_{\left\{\tau_{D}(X)>T\right\}},
$$

where $\tau_{D}(X)$ is the exit time of a domain $D \subset \mathbb{R}^{d}$ by a $d$-dimensional underlying asset $X=$ $\left(X^{1}, \ldots, X^{d}\right)$.

Here are some useful definitions.

Definition. The option is said to be an up-and-out option if it knocks out when the price of its underlying asset crosses a specified value. It is said a down-and-out option if it has barrier below the initial asset price and knocks out if the underlying asset price falls below the barrier.

The payoff of an up-and-out call expiring at time $T$, with strike price $K$ and up-and-out barrier $L$ is given by :

$$
\left(X_{T}-K\right)^{+} \mathbf{1}_{\left\{\sup _{t \in[0 . T]} X_{t} \leq L\right\}}
$$

and the payoff of a down-and-out call barrier option with maturity $T$, strike $K$ and barrier $L$ is given by

$$
\left(X_{T}-K\right)^{+} \mathbf{1}_{\left\{\inf _{t \in[0 . T]} X_{t} \geq L\right\}} \cdot
$$

The payoff of put options are defined similarly with $\left(K-X_{T}\right)^{+}$in place of $\left(X_{T}-K\right)^{+}$. 
Note that closed formulas are available for the price of such options in the Black-Scholes framework, see [6]. But this no longer holds when we move out from the Black-Scholes framework. So that we are led to estimate the prices by some numerical procedures. One of the used methods is the regular Brownian bridge method (see e.g. [1]). It provides approximation formulae of the price of barrier options using diffusion bridge methods. This leads to useful forms to approximate these prices from recursive formulas (already pointed out in [22]) similar to an algorithm used in [18] to estimate nonlinear filter by optimal quantization method. One difference of our setting with respect to the one of [18] is that our algorithm involves non-regular functions. Furthermore, if we consider local volatility model in the previous setting, one way of processing the algorithm is to use Lloyd's algorithm (or stochastic algorithms) to compute the optimal grids and the transition probabilities. But, because of the irregularity of functions appearing in our context, one must increase the grid sizes of the marginal quantization of the price process to obtain good approximations of the prices. It is clear that this will be very time consuming to use Lloyd's algorithm to compute grids sizes, and, this also depends to the parameters of the model. Moreover, the marginal quantized process is not a Markov chain and, for numerics, it is forced to satisfy the Markov property.

In this work, we propose a procedure based on (quadratic) marginal functional quantization method. It consists first in considering the ordinary differential equation (ODE) resulting to the substitution of the Brownian motion appearing in the dynamics of the price process 1.1 by one quadratic quantization of the Brownian motion. Then, constructing some "good" marginal quantization of the price process based on the solution of the previous ODE's, we show how to estimate the premium of barrier options from a recursive formula similar to an algorithm used to estimate nonlinear filter using optimal quantization method. Note that by construction, the marginal quantized discrete process is a Markov chain. Furthermore, because this procedure is based on the quantization of the Brownian motion, it does not depend on model parameters and price estimates are obtained in few seconds (at most in 6 seconds and sometimes instantaneously, for considered examples). Numerical simulations are performed in the Black-Scholes model and in the local volatility model called a pseudo CEV model. A comparison with the Regular Brownian Bridge method show that the former method may some times be faster and competitive with respect to the last one.

The paper is organized as follow. Since in a general setting, the estimation of the prices requires paths discretization of the process, we will recall in Section 2 the Euler scheme and some relevant convergence rate. Then, we will see in Section 3 how to derive the price estimates from (continuous) Euler scheme. The obtained formulas are well known and are moreover in a useful form to apply an algorithm similar to that used in nonlinear filtering estimation via optimal quantization. The algorithm and the relevant error are given in Section 4 This algorithm involves the marginal quantization of the stock price process and, in Section 5, we show how to construct such a process from a basic construction of functional quantization of a diffusion process. We end by some numerical experiments where we compare our method with the regular Brownian bridge method in the Black-Scholes model and in the pseudo CEV model.

\section{Euler Scheme}

Consider a one-dimensional Brownian diffusion process $\left(X_{t}\right)_{t \in[0, T]}$, solution of the following stochastic differential equation

$$
d X_{t}=b\left(X_{t}\right) d t+\sigma\left(X_{t}\right) d W_{t}, \quad X_{0}=x \in \mathbb{R}
$$


where $b: \mathbb{R} \rightarrow \mathbb{R}, \sigma: \mathbb{R} \rightarrow \mathbb{R}$ are continuous functions satisfying conditions (1.2), (1.3) and $\left(W_{t}\right)_{t \in[0, T]}$ denotes a one-dimensional Brownian motion defined on $(\Omega, \mathcal{F}, \mathbb{P})$.

Let us divide the set $[0, T]$ into $n$ subsets of length $T / n$ and set for every $k=0, \ldots, n, t_{k}=\frac{k T}{n}$. The stepwise constant Euler scheme is defined by

$$
\tilde{X}_{t_{k+1}}=\tilde{X}_{t_{k}}+b\left(\tilde{X}_{t_{k}}\right) \frac{T}{n}+\sigma\left(\tilde{X}_{t_{k}}\right) \sqrt{\frac{T}{n}} Z_{k+1}, \quad \tilde{X}_{0}=x, k=0, \ldots, n-1
$$

where $\left(Z_{k}\right)_{1 \leq k \leq n}$ is a sequence of $i . i . d$ random variables distributed as $\mathcal{N}(0 ; 1)$.

For every $t \in[0, T]$, set $\underline{t}=t_{k}$ if $t \in\left[t_{k}, t_{k+1}\right), \quad k=0, \ldots, n-1$. A natural extension of the discrete Euler scheme is the continuous Euler scheme defined for every $t \in[0, T]$ by

$$
\bar{X}_{t}=\bar{X}_{\underline{t}}+b\left(\bar{X}_{\underline{t}}\right)(t-\underline{t})+\sigma\left(\bar{X}_{\underline{t}}\right)\left(W_{t}-W_{\underline{t}}\right), \quad \bar{X}_{0}=x
$$

which satisfies the SDE

$$
\bar{X}_{t}=x+\int_{0}^{t} b\left(\bar{X}_{\underline{\mathbf{S}}}\right) d s+\int_{0}^{t} \sigma\left(\bar{X}_{\underline{\mathbf{S}}}\right) d W_{s} .
$$

The above paths discretization methods generate some errors which estimates are given in the following results (see e.g []).

$\triangleright$ Strong error rate. Assume $b$ and $\sigma$ satisfy for every $\alpha \in(0,1)$,

$$
\forall t \in[0, T], \forall y, z \in \mathbb{R}^{d}, \quad|b(s, y)-b(t, z)| \leq C\left(|t-s|^{\alpha}+|y-z|\right) .
$$

Then,

(a) for every $p>0$, for every $n \geq 1$,

$$
\left\|\sup _{t \in[0, T]}\left|X_{t}-\bar{X}_{t}\right|\right\|_{p} \leq C_{b, \sigma, p} e^{T C_{b, \sigma, p}}(1+|x|)\left(\frac{T}{n}\right)^{\frac{1}{2} \wedge \alpha}
$$

(b) for every $p>0$, for every $n \geq 1$,

$$
\left\|\sup _{t \in[0, T]}\left|X_{t}-\tilde{X}_{t}\right|\right\|_{p} \leq C_{b, \sigma, p} e^{T C_{b, \sigma, p}}(1+|x|) \sqrt{\frac{\log (n)}{n}} .
$$

$\triangleright$ Weak error. We recall some weak error estimates for path-dependent options (we refer e.g. [10] for the proofs). Let

$$
\mathbb{D}\left([0, T], \mathbb{R}^{d}\right):=\left\{\xi:[0, T] \rightarrow \mathbb{R}^{d}, \text { càdlàg }\right\} .
$$

If $F: \mathbb{D}\left([0, T], \mathbb{R}^{d}\right) \rightarrow \mathbb{R}$ is a Lipschitz functional for the sup norm, that is,

$$
\left|F(\xi)-F\left(\xi^{\prime}\right)\right| \leq C_{F} \sup _{t \in[0, T]}\left|\xi(t)-\xi^{\prime}(t)\right|
$$

then

$$
\left|\mathbb{E} F\left(\left(X_{t}\right)_{t \in[0, T]}\right)-\mathbb{E} F\left(\left(\bar{X}_{t}\right)_{t \in[0, T]}\right)\right| \leq \frac{C}{\sqrt{n}}
$$

and

$$
\left|\mathbb{E} F\left(\left(X_{t}\right)_{t \in[0, T]}\right)-\mathbb{E} F\left(\left(\tilde{X}_{t}\right)_{t \in[0, T]}\right)\right| \leq C \sqrt{\frac{\log n}{n}}
$$


On the other hand, if a domain $D$ has a smooth enough boundary, $b, \sigma \in \mathcal{C}^{3}(\mathbb{R})$ and $\sigma$ uniformly elliptic on $D: \exists \sigma_{0}>0, \forall x \in \mathbb{R} \sigma^{2}(x) \geq \sigma_{0}^{2}$, then, for every bounded measurable function $f$ satisfying $d(\operatorname{supp}(f), \partial D) \geq 2 \varepsilon>0$,

$$
\mathbb{E}\left(f(\bar{X}) \mathbf{1}_{\{\tau(\bar{X})>T\}}\right)-\mathbb{E}\left(f(X) \mathbf{1}_{\{\tau(X)>T\}}\right)=C n^{-1}+o\left(n^{-1}\right)
$$

and

$$
\mathbb{E}\left(f(\tilde{X}) \mathbf{1}_{\{\tau(\tilde{X})>T\}}\right)-\mathbb{E}\left(f(X) \mathbf{1}_{\{\tau(X)>T\}}\right)=O\left(n^{-1 / 2}\right)
$$

where $n$ is the number of discretization steps and $\tau(Y)$ is the exit time of the process $Y$ from the open set $D$, i.e

$$
\tau(Y)=\inf \left\{t \in[0, T], Y_{t} \in D^{c}\right\} .
$$

Then the convergence rate is of order $n^{-1}$ for the continuous Euler scheme and of order $n^{-1 / 2}$ for the discrete one.

\section{Approximation of knock out option prices using diffusion bridge}

According to the convergence rate for the continuous Euler scheme we would like to estimate the price of path-dependent options by replacing the asset price process $\left(X_{t}\right)_{t \in[0, T]}$ by its continuous Euler process $(\bar{X})_{t \in[0, T]}$ in (1.4). Then, given values of the process $\left(\bar{X}_{t}\right)$ at discrete times $t_{k}, k=0, \ldots, n$, one deduces formulas integrating the useful information which is the probability that the barrier is not knocked over the time interval $[0, T]$. Remark that this information is lost when replacing $X$ by the discrete Euler process $\tilde{X}$ in (1.4) because we do not known if whether or not the barrier is knocked between time intervals $\left(t_{k}, t_{k+1}\right), k=0, \ldots, n-1$. But, integrating this information in the former case requires the knowledge of the distributions of the maximum and the minimum of the continuous Euler process $\left(\bar{X}_{t}\right)$ over the time interval $[0, T]$, given its values at the discrete time observations $t_{k}$.

Proposition 3.1. We have

$$
\mathcal{L}\left(\max _{t \in[0, T]} \bar{X}_{t} \mid \bar{X}_{t_{k}}=x_{k}, k=0, \ldots, n\right)=\mathcal{L}\left(\max _{k=0, \ldots, n-1} G_{x_{k}, x_{k+1}}^{-1}\left(U_{k}\right)\right)
$$

and

$$
\mathcal{L}\left(\min _{t \in[0, T]} \bar{X}_{t} \mid \bar{X}_{t_{k}}=x_{k}, k=0, \ldots, n\right)=\mathcal{L}\left(\min _{k=0, \ldots, n-1} F_{x_{k}, x_{k+1}}^{-1}\left(U_{k}\right)\right)
$$

where $\left(U_{k}\right)_{k=0, \ldots, n-1}$ are i.i.d random variables uniformly distributed over the unit interval, $G_{x, y}^{-1}$ and $F_{x, y}^{-1}$ are the inverse functions of the conditional distribution functions $G_{x, y}$ and $F_{x, y}$ defined by

$$
G_{x, y}(u)=\left(1-e^{-2 n \frac{(x-u)(y-u)}{T \sigma^{2}(x)}}\right) \mathbf{1}_{\{u \geq \max (x, y)\}}
$$

and

$$
F_{x, y}(u)=1-\left(1-e^{-2 n \frac{(x-u)(y-u)}{T \sigma^{2}(x)}}\right) \mathbf{1}_{\{u \leq \min (x, y)\}}
$$

This result is proved using the independence property of the processes $\left(\bar{X}_{t}\right)_{t \in\left[t_{k}, t_{k+1}\right]}$, for $k=$ $0, \ldots, n-1$, given the $\bar{X}_{t_{k}}=x_{k}$, and the knowledge of the distribution of the supremum (and the infimum) of brownian bridge diffusion over time intervals $\left(t_{k}, t_{k+1}\right)$, with end points $x_{k}$ and $x_{k+1}$.

From the above proposition we deduce general formulas making a connexion between the expectation of a functional of both the terminal value $\bar{X}_{T}$ of the process $\left(\bar{X}_{t}\right)$ and its maximum (or the minimum) over the time interval $[0, T]$. From now on we make the abuse of notation $\bar{X}_{k}:=\bar{X}_{t_{k}}, \forall k \in$ $\{0, \ldots, n\}$. 
Proposition 3.2. (a) Let $f$ be a real-valued non negative function defined on $\mathbb{R}_{+}^{2}$ such that $f(x, \cdot)$ is a nonnegative function satisfying

$$
\sup _{x>0} \mathbb{E} f\left(x, \max _{t \in[0, T]} \bar{X}_{t}\right)<+\infty .
$$

Then

$$
\mathbb{E} f\left(\bar{X}_{T}, \max _{t \in[0, T]} \bar{X}_{t}\right)=\mathbb{E} f\left(\bar{X}_{T}, 0\right)+\mathbb{E} \int_{0}^{+\infty}\left(1-\prod_{k=1}^{n} G_{\bar{X}_{k-1}, \bar{X}_{k}}(z)\right) d_{z} f\left(\bar{X}_{T}, z\right) .
$$

Likewise if

$$
\left.\sup _{x>0} \mathbb{E} f\left(x, \min _{t \in[0, T]} \bar{X}_{t}\right)<+\infty\right)
$$

then

$$
\mathbb{E} f\left(\bar{X}_{T}, \min _{t \in[0, T]} \bar{X}_{t} \vee 0\right)=\mathbb{E} f\left(\bar{X}_{T}, 0\right)+\mathbb{E} \int_{0}^{+\infty}\left(\prod_{k=1}^{n}\left(1-F_{\bar{X}_{k-1}, \bar{X}_{k}}(z)\right)\right) d_{z} f\left(\bar{X}_{T}, z\right) .
$$

(b) If furthermore $f_{\infty}(x):=\lim _{y \rightarrow+\infty} f(x, y)<+\infty$ for every $x>0$. Then

$$
\mathbb{E} f\left(\bar{X}_{T}, \max _{t \in[0, T]} \bar{X}_{t}\right)=\mathbb{E} f_{\infty}\left(\bar{X}_{T}\right)-\mathbb{E} \int_{0}^{+\infty}\left(\prod_{k=1}^{n} G_{\bar{X}_{k-1}, \bar{X}_{k}}(z)\right) d_{z} f\left(\bar{X}_{T}, z\right)
$$

and

$$
\mathbb{E} f\left(\bar{X}_{T}, \min _{t \in[0, T]} \bar{X}_{t} \vee 0\right)=\mathbb{E} f_{\infty}\left(\bar{X}_{T}\right)-\mathbb{E} \int_{0}^{+\infty}\left(1-\prod_{k=1}^{n}\left(1-F_{\bar{X}_{k-1}, \bar{X}_{k}}(z)\right)\right) d_{z} f\left(\bar{X}_{T}, z\right) .
$$

This proposition follows from the following lemma.

Lemma 3.1. (a) Let $Z$ be a positive random variable and let $g$ be a nonnegative function with finite variation (on compact sets) such that

$$
\mathbb{E}\left(\int_{] 0, Z]}|d g|\right)<+\infty
$$

Then

$$
\mathbb{E} g(Z)=g(0)+\int_{(0,+\infty)} \mathbb{P}(Z \geq z) d g(z) .
$$

(b) If furthermore $g_{\infty}:=\lim _{x \rightarrow+\infty} g(x)<+\infty$ then

$$
\mathbb{E} g(Z)=g_{\infty}-\int_{(0,+\infty)} \mathbb{P}(Z<z) d g(z) .
$$


Proof. (a) We have

$$
g(Z)=g(0)+\int_{] 0, Z]} d g(u) .
$$

It follows that

$$
\begin{aligned}
\mathbb{E} g(Z) & =g(0)+\mathbb{E} \int_{] 0, Z]} d g(u) \\
& =g(0)+\int_{(0,+\infty)} \mathbb{P}(Z \geq z) d g(z),
\end{aligned}
$$

the last inequality coming from Fubini's theorem; which can be applied owing to assumption (3.8).

(b) Just use the fact that $\mathbb{P}(Z \geq u)=1-\mathbb{P}(Z<u)$.

Now we are in position to prove Proposition 3.2

Proof of Proposition 3.2 (b) One deduces from (3.1) that

$$
\mathbb{E}\left(f\left(x_{n}, \max _{t \in[0, T]} \bar{X}_{t}\right) \mid \bar{X}_{k}=x_{k}, k=0, \ldots, n\right)=\mathbb{E}\left(f\left(x_{n}, \max _{0 \leq k \leq n-1} G_{x_{k}, x_{k+1}}^{-1}\left(U_{k}\right)\right)\right)
$$

where $G_{x, y}^{-1}$ and the $U_{k}$ are defined like in (3.1). Then, applying Lemma 3.1 (b) to the function $g(z)=f\left(x_{n}, z\right)$ gives

$$
\begin{aligned}
\mathbb{E}\left(f\left(x_{n}, \max _{0 \leq k \leq n-1} G_{x_{k}, x_{k+1}}^{-1}\left(U_{k}\right)\right)\right) & =f_{\infty}\left(x_{n}\right)-\int_{0}^{+\infty} \mathbb{P}\left(\max _{0 \leq k \leq n-1} G_{x_{k}, x_{k+1}}^{-1}\left(U_{k}\right) \leq z\right) d_{z} f\left(x_{n}, z\right) \\
& =f_{\infty}\left(x_{n}\right)-\int_{0}^{+\infty}\left(\prod_{k=0}^{n-1} \mathbb{P}\left(U_{k} \leq G_{x_{k}, x_{k+1}}(z)\right)\right) d_{z} f\left(x_{n}, z\right) \\
& \left.=f_{\infty}\left(x_{n}\right)-\int_{0}^{+\infty}\left(\prod_{k=0}^{n-1} G_{x_{k}, x_{k+1}}(z)\right)\right) d_{z} f\left(x_{n}, z\right) .
\end{aligned}
$$

Consequently

$$
\begin{aligned}
\mathbb{E} f\left(\bar{X}_{T}, \max _{t \in[0, T]} \bar{X}_{t}\right) & =\mathbb{E}\left(\mathbb{E}\left(f\left(\bar{X}_{T}, \max _{t \in[0, T]} \bar{X}_{t}\right) \mid \bar{X}_{k}=x_{k}, k=0, \ldots, n\right)\right) \\
& =\mathbb{E} f_{\infty}\left(\bar{X}_{T}\right)-\mathbb{E} \int_{0}^{+\infty}\left(\prod_{k=1}^{n} G_{\bar{X}_{k-1}, \bar{X}_{k}}(z)\right) d_{z} f\left(\bar{X}_{T}, z\right) .
\end{aligned}
$$

The formula relative to the minimum is proved likewise by using (3.2) in place of (3.1).

(a) is proved like $(b)$ by using Lemma 3.1 (a) instead of Lemma 3.1 (b).

Proposition 3.2 allows us to rewrite the estimates of the premiums of some usual exotic options (in particular barrier options) in a useful form in view of the optimal quantization approximation method as well as of Monte Carlo simulation methods. Let us mention that the following representations of the price estimates of Barrier options are well known even if the computational method used here to derive them is a little different. 
Proposition 3.3. Let $f(x)=(x-K)^{+}$and $g(x)=(K-x)^{+}$.

(a) The price of an up-and-out put option expiring at time $T$ with strike $K$ and up-and-out barrier $L$ is estimated by

$$
\bar{P}_{U B}:=e^{-r T} \mathbb{E}\left(\left(K-\bar{X}_{T}\right)^{+} \mathbf{1}_{\left\{\sup _{t \in[0, T]} \bar{X}_{t} \leq L\right\}}\right)=e^{-r T} \mathbb{E}\left(g\left(\bar{X}_{T}\right) \prod_{k=1}^{n} G_{\bar{X}_{k-1}, \bar{X}_{k}}(L)\right) .
$$

(b) The price of an up-and-out call option expiring at time $T$ with strike $K$ and up-and-out barrier $L$ can be approximated by

$$
\bar{C}_{U B}:=e^{-r T} \mathbb{E}\left(\left(\bar{X}_{T}-K\right)^{+} \mathbf{1}_{\left\{\sup _{t \in[0, T]} \bar{X}_{t} \leq L\right\}}\right)=e^{-r T} \mathbb{E}\left(f\left(\bar{X}_{T}\right) \prod_{k=1}^{n} G_{\bar{X}_{k-1}, \bar{X}_{k}}(L)\right) .
$$

(c) The price of an down-and-out put option expiring at time $T$ with strike $K$ and down-and-out barrier $L$ can be approximated by

$$
\bar{P}_{O B}:=e^{-r T} \mathbb{E}\left(\left(K-\bar{X}_{T}\right)^{+} \mathbf{1}_{\left\{\inf _{t \in[0, T]} \bar{X}_{t} \geq L\right\}}\right)=e^{-r T} \mathbb{E}\left(g\left(\bar{X}_{T}\right) \prod_{k=1}^{n}\left(1-F_{\bar{X}_{k-1}, \bar{X}_{k}}(L)\right)\right) .
$$

(d) The price of an down-and-out call option expiring at time $T$ with strike $K$ and up-and-out barrier $L$ is approximated by the following formula :

$$
\bar{C}_{O B}:=e^{-r T} \mathbb{E}\left(\left(\bar{X}_{T}-K\right)^{+} \mathbf{1}_{\left\{\inf _{t \in[0, T]} \bar{X}_{t} \geq L\right\}}\right)=e^{-r T} \mathbb{E}\left(f\left(\bar{X}_{T}\right) \prod_{k=1}^{n}\left(1-F_{\bar{X}_{k-1}, \bar{X}_{k}}(L)\right)\right) .
$$

Note that the right hand side of Equations (3.11), (3.12), (3.13), 3.14) are obtained by re-conditioning. Then, it follows from Jensen inequality that the corresponding variances are smaller than the variances induced by the left hand side of the same equations.

Proof. (a) Let $f(x)=(x-K)^{+}, g(z)=\mathbf{1}_{\{z \leq L\}}$ and set $h(x, z)=f(x) g(z)$. Then it follows from (3.6) that

$$
\mathbb{E}\left(\left(\bar{X}_{T}-K\right)^{+} \mathbf{1}_{\left\{\sup _{t \in[0, T]} \bar{X}_{t} \leq L\right\}}\right)=\mathbb{E} h_{\infty}\left(\bar{X}_{T}\right)-\mathbb{E} \int_{0}^{+\infty}\left(f\left(\bar{X}_{T}\right) \prod_{k=1}^{n} G_{\bar{X}_{k-1}, \bar{X}_{k}}(z)\right) d g(z) .
$$

Now $\forall x \geq 0, h_{\infty}(x)=0$ and $d g(z)=-\delta_{L}(z)$. Then

$$
C_{U B}=e^{-r T} \mathbb{E}\left(f\left(\bar{X}_{T}\right) \prod_{k=1}^{n} G_{\bar{X}_{k-1}, \bar{X}_{k}}(L)\right) .
$$

The items $(b),(c),(d)$ are proved in the same way as $(a)$.

In the next section, we show how to estimate the previous prices by optimal quantization. We will give first an approximating algorithm and then, the induced error. Since this algorithm involves the quantization of the price process and its transition probabilities, we will point out how to construct a functional quantization of the price process and how to estimate its transition probabilities. 


\section{Estimation of the prices by marginal quantization}

The aim of this section is to propose an algorithm based on optimal quantization to compute the path dependent options pointed out in Section 3. To this end we will approximate by optimal quantizations some expressions of the form

$$
V:=\mathbb{E}\left(f\left(\bar{X}_{n}\right) \prod_{k=1}^{n} g_{k}\left(\bar{X}_{k-1}, \bar{X}_{k}\right)\right)
$$

where $f$ is a bounded measurable function on $\mathbb{R}^{d}$ taking values on $\mathbb{R}$ and $g_{k}(\cdot, \cdot)$ a measurable function on $\mathbb{R}^{d} \times \mathbb{R}^{d}$ which may depend on some real parameters like for Barrier options where it depends also on the barrier.

\subsection{The algorithm}

We will mainly refer to [18], where numerical solving of nonlinear filtering with discrete-time observation have been performed by optimal quantization methods. The only change is that in our setting we will drop the dependance on the noisy observations (i.e the $Y_{k}$ 's following the notations in [18]) because our problem of interest here is not a filtering problem.

We define for any $k=1, \ldots, n$, the bounded transition kernel $H_{k}$ by

$$
H_{k} f(x)=\mathbb{E}\left(f\left(\bar{X}_{k}\right) g_{k}\left(x, \bar{X}_{k}\right) \mid \bar{X}_{k-1}=x\right)=\int f(y) g_{k}(x, y) P_{k}(x, d y)
$$

where $P_{k}(x, \cdot)=\mathcal{L}\left(\bar{X}_{k}=\cdot \mid \bar{X}_{k-1}=x\right)$. For convenience, we set

$$
H_{0} f(x)=\mathbb{E}\left(f\left(\bar{X}_{0}\right)\right)=\int f(x) \mu(d x) .
$$

Now for any $k \in\{1, \ldots, n\}$ set

$$
\pi_{k} f=\mathbb{E}\left(f\left(\bar{X}_{k}\right) \prod_{i=1}^{k} g_{k}\left(\bar{X}_{i-1}, \bar{X}_{i}\right)\right) .
$$

We have

$$
\begin{aligned}
\pi_{k} f & =\mathbb{E}\left(\mathbb{E}\left(f\left(\bar{X}_{k}\right) \prod_{i=1}^{k} g_{i}\left(\bar{X}_{i-1}, \bar{X}_{i}\right) \mid \mathcal{F}_{t_{k-1}}\right)\right) \\
& =\mathbb{E}\left(\mathbb{E}\left(f\left(\bar{X}_{k}\right) g_{k}\left(\bar{X}_{k-1}, \bar{X}_{k}\right) \mid \bar{X}_{k-1}\right) \prod_{i=1}^{k-1} g_{i}\left(\bar{X}_{i-1}, \bar{X}_{i}\right)\right) \\
& =\mathbb{E}\left(H_{k}\left(f\left(\bar{X}_{k-1}\right)\right) \prod_{i=1}^{k-1} g_{i}\left(\bar{X}_{i-1}, \bar{X}_{i}\right)\right)
\end{aligned}
$$

It follows that

$$
\pi_{k} f=\pi_{k-1} H_{k} f, \quad k=1, \ldots, n
$$

so that

$$
V=\pi_{n} f=\left(H_{0} \circ H_{1} \circ \cdots \circ H_{n}\right) f .
$$


Then, to estimate $V$ we need to approximate $\pi_{n}$. At this step, suppose that we have access to the quantization $(\widehat{X})_{t_{k}}$ of the price process over the time steps $t_{k}, k=0, \ldots, n$ on grids $\Gamma_{k}=$ $\left\{x_{k}^{1}, \ldots, x_{k}^{N_{k}}\right\}$ of sizes $N_{k}, k=0, \ldots, n$ (see further on for facts about quantization).

Owing to equation (4.4) our aim is to estimate the price using an approximation of the probability transition $P_{k}\left(x_{k}, d x_{k+1}\right)$ of $\bar{X}_{k+1}$ given $\bar{X}_{k}$. These probability transitions are approximated by the probability transition matrix $\hat{p}_{k}:=\left(\hat{p}_{k}^{i j}\right)$ of $\widehat{X}_{k+1}$ given $\widehat{X}_{k}$ :

$$
\hat{p}_{k}^{i j}=\mathbb{P}\left(\widehat{X}_{k}=x_{k}^{j} \mid \widehat{X}_{k-1}=x_{k-1}^{i}\right) ; i=1, \ldots, N_{k-1}, j=1, \ldots, N_{k} .
$$

Then, following Equation (4.2), we estimate the transition kernel matrix $H_{k}$ by the quantized transition kernel $\widehat{H}_{k}$ given by

$$
\widehat{H}_{k}=\sum_{j=1}^{N_{k}} \widehat{H}_{k}^{i j} \delta_{x_{k-1}^{i}}, \quad k=1, \ldots, n
$$

where

$$
\widehat{H}_{k}^{i j}=g_{k}\left(x_{k-1}^{i}, x_{k}^{j}\right) \hat{p}_{k}^{i j}, \quad i=1, \ldots, N_{k-1}, j=1, \ldots, N_{k} .
$$

For $k=0$, we set (owing to (4.3) and to the fact that $X_{0}=x_{0}$ is not random)

$$
\widehat{H}_{0}=\delta_{x_{0}} .
$$

We finally approximate $\pi_{n}$ by

$$
\widehat{\pi}_{n}=\widehat{H}_{0} \circ \widehat{H}_{1} \circ \cdots \circ \widehat{H}_{n} ;
$$

which in turn can be computed by the forward induction

$$
\widehat{\pi}_{0}=\widehat{H}_{0}, \quad \widehat{\pi}_{k}=\widehat{\pi}_{k-1} \widehat{H}_{k}, \quad k=1, \ldots, n .
$$

It follows that the price $V=\pi_{n} f$ may be estimated by summery

$$
\widehat{V}:=\widehat{\pi}_{n} f .
$$

From the previous approach, we deduce the following estimations for options of interest using optimal functional quantization method. Set in this scope $f(x):=(x-K)^{+}$and $g(x):=(K-x)^{+}$.

$\triangleright$ Up-and-out options. According to the forgoing we estimate the price of an up-and-out put option by

$$
\widehat{P}_{U B}:=e^{-r T} \widehat{\pi}_{n} g
$$

and the price of up-and-out call option is approximated by

$$
\widehat{C}_{U B}:=e^{-r T} \widehat{\pi}_{n} f
$$

where $\widehat{\pi}_{n}$ is defined as in (4.8) with the associated transition kernel

$$
\widehat{H}_{k}^{i j}=G_{x_{k-1}^{i}, x_{k}^{j}}(L) \hat{p}_{k}^{i j}, \quad i=1, \ldots, N_{k-1} ; j=1, \ldots, N_{k} .
$$

$\triangleright$ Down-and-out options. The down-and-out put option's price is estimated by

$$
\widehat{P}_{O B}:=e^{-r T} \widehat{\pi}_{n} g
$$


and the price of down-and-out barrier call option is estimated by

$$
\widehat{C}_{O B}:=e^{-r T} \widehat{\pi}_{n} f
$$

where for both cases $\widehat{\pi}_{n}$ is defined as in (4.8) with the associated transition kernel

$$
\widehat{H}_{k}^{i j}=F_{x_{k-1}^{i}, x_{k}^{j}}(L) \hat{p}_{k}^{i j}, \quad i=1, \ldots, N_{k-1} ; j=1, \ldots, N_{k} .
$$

Remark 4.1. One numerical advantage of this algorithm is that $\widehat{\pi}_{n}$ does not depend on the function $f$ appearing in (4.5). Then, once $\widehat{\pi}_{n}$ is computed we deduce both the call and the put price approximations. On the other hand, considering Equation 4.7) one notices that as soon as $\widehat{X}_{k-1}$ reaches the barrier (for example, for the up-and-out option: there exists $i_{0}$ such that $x_{k-1}^{i_{0}}>L$ ), then, $\widehat{H}_{k}^{i j}=0$ for every $i \geq i_{0}$. For numerical computation, we may take account of this fact to reduce the computation time.

\subsection{Error analysis}

In order to have some upper bound of the quantization error estimate of $\pi f$ we need the following assumptions (A1) and (A2) :

(A1) The transition operator $P_{k}(x, d y)$ of $X_{k}$ given $X_{k-1}, k=1, \ldots, n$ are Lipschitz.

Recall that a probability transition $P$ on $\mathbb{R}^{d}$ is C-Lipschitz (with $\mathrm{C}>0$ ) if for any Lipschitz function $f$ on $\mathbb{R}^{d}$ with ratio $[f]_{\text {Lip }}, P f$ is Lipschitz with ratio $[P f]_{\text {Lip }} \leq \mathrm{C}[f]_{\text {Lip }}$. Then, one may define the Lipschitz ratio $[P]_{\text {Lip }}$ by

$$
[P]_{\text {Lip }}=\sup \left\{\frac{[P f]_{\text {Lip }}}{[f]_{\text {Lip }}}, f \text { a nonzero Lipschitz function }\right\}<+\infty .
$$

Then if the transition operators $P_{k}(x, d y), k=1, \ldots, n$ are Lipschitz, it follows that

$$
[P]_{\text {Lip }}:=\max _{k=1, \ldots, n}\left[P_{k}\right]_{\text {Lip }}<+\infty .
$$

(A2) It consists on the following two assumptions.

(i) For every $k=1, \ldots, n$, the functions $g_{k}(\cdot, \cdot)$ are bounded on $\mathbb{R}^{d} \times \mathbb{R}^{d}$ and we set

$$
\mathrm{K}_{g}:=\max _{k=1, \ldots, n}\left\|g_{k}\right\|_{\infty}
$$

(ii) For every $k=1, \ldots, n$, there exist two constants $\left[g_{k}^{1}\right]_{\text {Lip }}$ and $\left[g_{k}^{2}\right]_{\text {Lip }}$ so that for every $x, x^{\prime}, \widehat{x}, \widehat{x}^{\prime} \in$ $\mathbb{R}^{d}$

$$
\left|g_{k}\left(x, x^{\prime}\right)-g_{k}\left(\widehat{x}, \widehat{x}^{\prime}\right)\right| \leq\left[g_{k}^{1}\right]_{\text {Lip }}|x-\widehat{x}|+\left[g_{k}^{2}\right]_{\text {Lip }}\left|x^{\prime}-\widehat{x}^{\prime}\right| .
$$

Theorem 4.1. Under Assumptions (A1) and (A2) we have for every bounded Lipschitz continuous function $f$ on $\mathbb{R}^{d}$ and for every $p \geq 1$,

$$
\left|\pi_{n} f-\widehat{\pi}_{n} f\right| \leq \sum_{k=0}^{n} \mathrm{C}_{k}^{n}(f, p)\left\|X_{k}-\widehat{X}_{k}\right\|_{p}
$$

with

$$
\mathrm{C}_{k}^{n}(f, p)=\left(2-\delta_{2, p}\right) \mathrm{K}_{g}^{k}\left[u_{k}\right]_{L i p}+\mathrm{K}_{g}^{n-1}\|f\|_{\infty}\left(\left[g_{k+1}^{1}\right]_{L i p}+\left[g_{k+1}^{2}\right]_{L i p}\right)
$$


Proof. The proof follows from the proof of Theorem 3.1 in [18] by dropping the dependency on the noisy observations $\left(y_{1}, \ldots, y_{n}\right)$ following the notations of the authors.

Now, let us come back to the construction of the quantized price process $\left(\widehat{X}_{k}\right)_{k=0, \ldots, n}$. We show in the next section how to construct this process after making a short background on product functional quantization of gaussian processes, in particular, of brownian motion.

\section{Marginal functional quantization of the price process}

Before dealing with the construction of the marginal functional quantization of the price process, we make some background on functional quantization of gaussian processes.

\subsection{A brief overview on functional product quantization of gaussian processes}

We remind first some basic notions about optimal vector quantization. It is a process of approximating a continuous range of values or a very large set of discrete values by a relatively small set of discrete values. Rigorously speaking, the $L^{r}$-optimal quantization problem at level $n$ for a $\mathbb{R}^{d}$-valued random vector $X$ lying in $L^{r}(\Omega, \mathcal{A}, \mathbb{P})$ consists in finding the best approximation of $X$ by a Borel function of $X$ taking at most $n$ values. This problem can be reads as

$$
\begin{aligned}
e_{n, r}(X) & =\inf \left\{\left\|X-\widehat{X}^{\alpha}\right\|_{r}, \alpha \subset \mathbb{R}^{d}, \operatorname{card}(\alpha) \leq n\right\} \\
& =\inf _{\substack{\alpha \subset \mathbb{R}^{d} \\
\operatorname{card}(\alpha) \leq n}}\left(\int_{\mathbb{R}^{d}} d(x, \alpha)^{r} d P(x)\right)^{1 / r} .
\end{aligned}
$$

where $\widehat{X}^{\alpha}=\sum_{a \in \alpha} a \mathbf{1}_{\left\{X \in C_{a}(\alpha)\right\}}$ is the quantization of $X$ on the grid $\alpha$ and $\left(C_{a}(\alpha)\right)_{a \in \alpha}$ corresponds to a Voronoi tessellation of $\mathbb{R}^{d}$ (with respect to a norm $|\cdot|$ on $\mathbb{R}^{d}$ ), that is, a Borel partition of $\mathbb{R}^{d}$ satisfying for every $a \in \alpha$,

$$
C_{a}(\alpha) \subset\left\{x \in \mathbb{R}^{d}:|x-a|=\min _{b \in \alpha}|x-b|\right\} .
$$

The quantity $e_{n, r}(X)$ is called the $L^{r}$-mean quantization error. This error decreases to zero at a $n^{-1 / d}$-rate as the size $n$ of the codebook $\alpha$ goes to infinity. This convergence rate has been investigated in [4] and [23] for absolutely continuous probability measures under the quadratic norm on $\mathbb{R}^{d}$ and studied in great details in [11] under an arbitrary norm on $\mathbb{R}^{d}$ for absolutely continuous measures and some singular measures. Very recently, optimal vector quantization has become a promising tool in Numerical Probability owing to its ability to approximate either expectations or more significantly conditional expectations from some cubature formulas. This faculty to approximate conditional expectations is the crucial property used to solve some problems emerging in finance as optimal stopping problems (pricing and hedging American style options, see [2, 20], stochastic control problems (see [7, 19]) for portfolio management, nonlinear filtering problems (see [18, 21] and [5] for an application to credit risk).

A rigorous extension of optimal vector quantization to functional quantization is done in [14] where the vector quantization problem is transposed to random variables taking values in an infinite dimensional Hilbert space, in particular, to stochastic processes $\left(X_{t}\right)_{t \in[0,1]}$ viewed as random variables with values in $L^{2}([0,1], d t)$. Many others works have been done in this direction as e.g. [8]. From the numerical point of view, it is pointed out in [20] how a Gaussian process can be quantized using 
Karhunen-Loève product quantization based on product quantization of Gaussian random variables coming from the Karhunen-Loève expansion of the given Gaussian process. A closed formula for the distribution of the quantization of the Gaussian process (in particular for Brownian motion) is derived and some applications has been successfully performed in Finance, namely, in the pricing of vanilla and Asian call options in Heston model.

To recall some basic results about functional quantization suppose that $\left(H,(\cdot \mid \cdot)_{H}\right)$ is a separable Hilbert space and let $X:(\Omega, \mathcal{A}, \mathbb{P}) \mapsto H$ be square integrable $H$-valued random vector with distribution $\mathbb{P}_{X}$ defined on $(H, \mathcal{B}$ or $(H))$ where $\mathcal{B}$ or $(H)$ stands for the Borel $\sigma$-field. Let $\|\cdot\|$ denotes the $L_{H}^{2}(\Omega, \mathbb{P})$-norm defined by $\|X\|_{2}^{2}=\mathbb{E}\left(|X|_{H}^{2}\right)$.

Let $x:=\left\{x_{1}, \ldots, x_{n}\right\} \in H^{n}$ be an $n$-quantizer and let $\widehat{X}^{x}$ be the quantization of $X$ on the grid $x$ defined previously, where the Voronoi tessellation $\left(C_{i}(x)\right)_{1 \leq i \leq n}$ induced by $x$ satisfies for every $i \in\{1, \ldots, n\}$,

$$
C_{i}(x) \subset\left\{y \in H:\left|x_{i}-y\right|_{H}=\min _{1 \leq j \leq n}\left|x_{j}-y\right|_{H}\right\} .
$$

The quadratic quantization problem consists of finding an optimal quantizer $x \in H^{n}$ (if any), means, an $n$-quantizer which minimizes the quantization error $\left\|X-\widehat{X}^{x}\right\|_{2}$ over $H^{n}$. From the numerical integration viewpoint, finding an optimal quantization may be a difficult problem and we are sometimes let to find some 'good' quantization $\widehat{X}^{x}$ which is close to $X$ in distribution, so that for every Borel function $F: H \mapsto \mathbb{R}$, we can approximate $\mathbb{E} F(X)$ by

$$
\mathbb{E} F\left(\widehat{X}^{x}\right)=\sum_{i=1}^{n} F\left(x_{i}\right) \mathbb{P}_{X}\left(C_{i}(x)\right) .
$$

Then if we have access to both the $n$-quantizer $x=\left\{x_{1}, \ldots, x_{n}\right\}$ and the distribution associated to $\widehat{X}^{x},\left(\mathbb{P}_{X}\left(C_{i}(x)\right)\right)_{1<i<n}$, the estimation of $\mathbb{E} F(X)$ using Equation (5.2) is straightforward. The induced error depends on the regularity of the functional $F$ and here is some error bounds. Suppose that $X \in L_{H}^{2}(\Omega, \mathbb{P})$ and let $F$ be a Borel functional defined on $H$.

1. If $F$ is Lipschitz continuous with Lipschitz constant $[F]_{\text {Lip }}$ then for every $n$-quantizer $x$,

$$
\left|\mathbb{E} F(X)-\mathbb{E} F\left(\widehat{X}^{x}\right)\right| \leq[F]_{\text {Lip }}\left\|X-\widehat{X}^{x}\right\|_{2}
$$

so that if $\left(x_{n}\right)_{n \geq 1}$ is a sequence of quantizers satisfying $\lim _{n \rightarrow \infty}\left\|X-\widehat{X}^{x_{n}}\right\|_{2}=0$, then $\widehat{X}^{x_{n}}$ converge in distribution to $X$.

2. If $F$ is differentiable on $H$ with an $\theta$-Hölder differential $D F, \theta \in(0,1]$, then for every optimal $n$-quantizer $x$,

$$
\left|\mathbb{E} F(X)-\mathbb{E} F\left(\widehat{X}^{x}\right)\right| \leq[D F]_{\theta}\left\|X-\widehat{X}^{x}\right\|_{2}^{1+\theta} .
$$

Some others bound are available (we refer to [20] for more detail). Now let us say how to get some 'good' quantizers for Gaussian processes to make sense the previous errors bounds. We consider here a centered one-dimensional $L_{T}^{2}:=L^{2}([0, T], d t)$-valued Gaussian process $X$ satisfying

$$
\mathbb{E}|X|_{L_{T}^{2}}^{2}=\int_{0}^{T} \mathbb{E}\left(X_{s}^{2}\right) d s<+\infty .
$$

The process $X$ admits the following representation in the Karhunen-Loève basis (see e.g. [14])

$$
X(\omega) \stackrel{L_{T}^{2}}{=} \sum_{k \geq 1} \sqrt{\lambda_{k}} \xi_{k}(\omega) e_{k}^{X} \quad \mathbb{P}(d \omega)-\text { a.s. }
$$


where the sequence $\left(\xi_{k}\right)_{k \geq 1}$ defined for every $k \geq 1$ by

$$
\xi_{k}=\frac{\left(X \mid e_{k}^{X}\right)}{\sqrt{\operatorname{Var}\left(\left(X \mid e_{k}^{X}\right)\right)}}
$$

is a sequence of i.i.d $\mathcal{N}(0 ; 1)$-distributed random variables. Owing to the expansion (5.4), a natural way to produce a functional product quantization of a Gaussian process in $L_{T}^{2}$ of size at most $N$ is to use a product quantizer of the form

$$
\widehat{X}_{t}^{\left(d_{N}\right)}=\sum_{k=1}^{L} \sqrt{\lambda_{k}} \widehat{\xi}_{k}^{\left(N_{k}\right)} e_{k}^{X}(t)
$$

where $\widehat{\xi}_{k}^{x^{\left(N_{k}\right)}}$ is an optimal $N_{k}$-quantization of $\xi_{k}$ and $d_{N}:=N_{1} \times \cdots \times N_{L} \leq N$, with $N_{1}, \ldots, N_{L} \geq$ 2. An quadratic optimal product $N$-quantizers also noted $\widehat{X}_{t}^{\left(d_{N}\right)}$ is obtained by solving the optimization problem (see [14] for more detail):

$$
\min \left\{\left\|X-\widehat{X}^{\left(d_{N}\right)}\right\|_{2}, d_{N}=N_{1} \times \cdots \times N_{L} \leq N ; N_{1}, \ldots, N_{L} \geq 2 ; L \geq 1\right\} .
$$

We suppose from now on that the previous optimization problem can be solved, at least numerically, and that the optimal $L$-tuple still be denoted by $N_{1}, \ldots, N_{L}$. Then, numerical computation of a Gaussian process $X$ is possible as soon as we have numerical access to the eigensystem $\left(e_{n}^{X}, \lambda_{n}\right)$, which, for the Brownian motion $\left(W_{t}\right)_{t \in[0, T]}$, has a closed formula:

$$
e_{k}^{W}(t):=\sqrt{\frac{2}{T}} \sin \left(\pi(k-1 / 2) \frac{t}{T}\right) \quad \text { and } \quad \lambda_{k}:=\left(\frac{T}{\pi(k-1 / 2)}\right)^{2}, k \geq 1 .
$$

So, the one-dimensional quadratic optimal product quantizer $\alpha^{N}$, at level $N$, of the Brownian motion $\left(W_{t}\right)_{t \in[0, T]}$, is defined by

$$
\alpha_{i_{1}, \ldots, i_{L}}^{N}(t)=\sqrt{\frac{2}{T}} \sum_{k=1}^{L} \frac{T}{\pi(k-1 / 2)} \sin \left(\pi(k-1 / 2) \frac{t}{T}\right) x_{i_{k}}^{\left(N_{k}\right)}, 1 \leq i_{k} \leq N_{k}, 1 \leq k \leq L,
$$

where $x^{\left(N_{k}\right)}=\left\{x_{1}^{N_{k}}, \ldots, x_{N_{k}}^{N_{k}}\right\}$ is the optimal quantization of the $\mathcal{N}(0 ; 1)$ of size $N_{k}$ and $d_{N}=$ $\prod_{k=1}^{L} N_{k}$ is an optimal integer solving Problem (5.6) (with respect to the Brownian motion). Remark that for every $t \in[0, T]$, the marginal quantizer $\alpha_{i_{1}, \ldots, i_{L}}^{N}(t)$ is of size $d_{N}$. For numerics, a whole package of product $N$-quantizers of the standard Brownian motion are available at www . quantize. maths-fi.com. We move now to the construction of the quantized price process.

\subsection{Marginal functional quantization and transition probabilities}

Recall that the continuous Euler price process evolves following the SDE

$$
d X_{t}=b\left(X_{\underline{t}}\right) d t+\sigma\left(X_{\underline{t}}\right) d W_{t}, \quad X_{0}=x .
$$

Let $\left(\alpha^{N}\right)_{N \geq 1}$, with for every $N \geq 1, \alpha^{N}(t)=\left\{\alpha_{1}^{N}(t), \ldots, \alpha_{d_{N}}^{N}(t)\right\}$, be a sequence of optimal product $N$-quantizers of the Brownian motion and let

$$
\widehat{W}_{t}^{\left(d_{N}\right)}=\sum_{m=1}^{d_{N}} \alpha_{m}^{N}(t) \mathbf{1}_{\left\{W_{t} \in C_{m}\left(\alpha^{N}(t)\right)\right\}}, \quad t \in[0, T],
$$


be the marginal functional quantization of the Brownian motion. It is known that the sequence $\left(\alpha^{N}\right)_{N \geq 1}$ is rate-optimal, means,

$$
\left\|W-\widehat{W}^{\alpha^{N}}\right\|_{2}=O\left((\log N)^{-1 / 2}\right) .
$$

Consider the sequence $x^{N}=\left(x_{m}^{N}\right)_{m=1, \ldots, d_{N}}, N \geq 1$ of solutions of the ODE's

$$
x_{m}^{N}(t)=x+\int_{0}^{t}\left[b\left(x_{m}^{N}(\underline{s})\right)-\frac{1}{2} \sigma \sigma^{\prime}\left(x_{m}^{N}(\underline{s})\right)\right] d s+\int_{0}^{t} \sigma\left(x_{m}^{N}(\underline{s})\right) d \alpha_{m}^{N}(s), \quad m=1, \ldots, d_{N}
$$

$\underline{t}=t_{k}$ if $t \in\left[t_{k}, t_{k+1}\right), k=0, \ldots, n-1$, and define the marginal functional quantization of $\bar{X}_{t}$ over the grid $x^{N}(t)=\left\{x_{1}^{N}(t), \ldots, x_{d_{N}}^{N}(t)\right\}$ by

$$
\widehat{X}_{t}^{N}=\sum_{m=1}^{d_{N}} x_{m}^{N}(t) \mathbf{1}_{\left\{\bar{X}_{t} \in C_{m}\left(x^{N}(t)\right)\right\}},
$$

so that Equation (5.8) can be written as

$$
\widehat{X}_{t}^{N}=x+\int_{0}^{t}\left[b\left(\widehat{X}_{\underline{s}}^{N}\right)-\frac{1}{2} \sigma \sigma^{\prime}\left(\widehat{X}_{\underline{s}}^{N}\right)\right] d s+\int_{0}^{t} \sigma\left(\widehat{X}_{\underline{s}}^{N}\right) d \widehat{W}_{s}^{\left(d_{N}\right)} .
$$

Recall that the process $\left(\bar{X}_{t_{k}}\right)$ is a Markov chain. Then, since by construction $\sigma\left(\bar{X}_{t_{k}}, k=0, \ldots, n\right)=$ $\sigma\left(\widehat{X}_{t_{k}}^{N}, k=0, \ldots, n\right)$, the discrete process $\left(\widehat{X}_{t_{k}}^{N}\right)_{k=0, \ldots, n}$ is a Markov chain. On the other hand, since the additional term $\frac{1}{2} \sigma \sigma^{\prime}$ appears in the ODE (this correction term can be dropped by considering the stochastic integral in (5.7) in the sense of Stratonovich integral for $L_{L_{T}^{2}}^{p}(\Omega, \mathbb{P})$ convergence investigation tools, see [14]), we must make the supplementary assumption that $\sigma$ is continuously differentiable with bounded derivative to guaranty the existence and the uniqueness of the solution. Now, given the quantization process $\left(\widehat{X}^{N}\right)$, to complete the estimation of the price of barrier options following the introduced algorithm in Section 4.1 it suffice to compute the transition probabilities appearing in (4.6). Notice that all our grids $x^{N}\left(t_{k}\right)$ are of size $d_{N}=N_{1} \times \ldots N_{L}$. To define correctly the Voronoi cell associated to the grids $x^{N}\left(t_{k}\right)$ we will consider that for every time step $t_{k}$, $x^{N}\left(t_{k}\right)=\left\{x_{1}^{N}\left(t_{k}\right), \ldots, x_{d_{N}}^{N}\left(t_{k}\right)\right\}$ is a descendent ordered set. The computation of the transition probabilities will be made differently according to the following two situations.

$\triangleright$ The cumulative distribution function $F(\cdot ; x)$ of the conditional law of $X_{t}$ given $X_{s}=x$ is known for every $s \leq t$. For example, this is the case in the Black Scholes model where $F(\cdot ; x)$ is the cumulative distribution function of the lognormal distribution. In this case, since following Equation (5.9), $\sigma\left(\bar{X}_{t_{k}}, k=0, \ldots, n\right)=\sigma\left(\widehat{X}_{t_{k}}^{N}, k=0, \ldots, n\right)$, the probabilities are estimated by

$$
\hat{p}_{k}^{i j} \approx F\left(x_{j+}^{N}\left(t_{k}\right) ; x_{i}^{N}\left(t_{k-1}\right)\right)-F\left(x_{j-}^{N}\left(t_{k}\right) ; x_{i}^{N}\left(t_{k-1}\right)\right),
$$

with for every $k=0, \ldots, n-1$,

$$
\left\{\begin{array}{l}
x_{j+}^{N}\left(t_{k}\right):=\frac{x_{j}^{N}\left(t_{k}\right)+x_{j+1}^{N}\left(t_{k}\right)}{2} ; x_{j-}^{N}\left(t_{k}\right):=\frac{x_{j}^{N}\left(t_{k}\right)+x_{j-1}^{N}\left(t_{k}\right)}{2} ; j=1, \ldots, d_{N}-1 ; \\
x_{1-}^{N}\left(t_{k}\right)=0 ; x_{d_{N}^{+}}^{N}\left(t_{k}\right)=+\infty .
\end{array}\right.
$$

In fact, we have for every $k=0, \ldots, n-1$,

$$
\begin{aligned}
\hat{p}_{k}^{i j} & =P\left(\bar{X}_{k} \in C\left(x^{N}\left(t_{k}\right)\right) \mid \bar{X}_{k-1} \in C\left(x^{N}\left(t_{k-1}\right)\right)\right) \\
& \approx P\left(X_{k+1} \in C_{j}\left(x^{N}\left(t_{k}\right)\right) \mid X_{k-1} \in C_{i}\left(x^{N}\left(t_{k-1}\right)\right)\right) \\
& =P\left(X_{k} \leq x_{j+}^{N}\left(t_{k}\right) \mid X_{k-1} \in C_{i}\left(x^{N}\left(t_{k-1}\right)\right)\right)-P\left(X_{k} \leq x_{j-}^{N}\left(t_{k}\right) \mid X_{k-1} \in C_{i}\left(x^{N}\left(t_{k-1}\right)\right)\right) .
\end{aligned}
$$


Afterward, we have for every $z \geq 0$,

$$
P\left(X_{k} \leq z \mid X_{k-1} \in C_{i}\left(x^{N}\left(t_{k-1}\right)\right)\right)=\frac{P\left(X_{k} \leq z ; X_{k-1} \in C_{i}\left(x^{N}\left(t_{k-1}\right)\right)\right)}{P\left(X_{k-1} \in C_{i}\left(x^{N}\left(t_{k-1}\right)\right)\right)},
$$

and considering the numerator in the right hand side of the previous equation we have

$$
\begin{aligned}
P\left(X_{k} \leq z ; X_{k-1} \in C_{i}\left(x^{N}\left(t_{k-1}\right)\right)\right) & =\int_{-\infty}^{z}\left(\int_{C_{i}\left(x^{N}\left(t_{k-1}\right)\right)} P\left(X_{k} \in d x \mid X_{k-1}=y\right) d P_{X_{k-1}}(y)\right) d x \\
& =\int_{C_{i}\left(x^{N}\left(t_{k-1}\right)\right)} F(z ; y) d P_{X_{k-1}}(y) \\
& \approx F\left(z ; x_{i}^{N}\left(t_{k-1}\right)\right) P\left(X_{k-1} \in C_{i}\left(x^{N}\left(t_{k-1}\right)\right)\right) .
\end{aligned}
$$

The last quantity is the approximation of the right hand side of (5.11) by optimal quantization with one grid's point, considering that $\left\{x_{i}^{N}\left(t_{k-1}\right)\right\}$ is the quantizer of size one of the random variable $\bar{X}_{t_{k-1}}$ over the Voronoi cell $C_{i}\left(\left(x^{N}\left(t_{k-1}\right)\right)\right.$.

$\triangleright$ The cumulative distribution function $F(\cdot, x)$ of the conditional law of $X_{t}$ given $X_{s}=x$ is unknown. In this case, considering the (discrete) Euler Scheme of the price process (see (2.2) we estimate $F$ by the cumulative distribution function $\tilde{F}$ of the $\mathcal{N}\left(m_{k} ; \sigma_{k}^{2}\right)$ with

$$
m_{k}=\widehat{X}_{k-1}+b\left(t_{k}, \widehat{X}_{k-1}\right) \frac{T}{n} ; \quad \sigma_{k}^{2}=\sigma^{2}\left(t_{k}, \widehat{X}_{k-1}\right) \frac{T}{n},
$$

so that for every $i, j=1, \ldots, d_{N}$,

$$
\hat{p}_{k}^{i j} \approx \tilde{F}\left(x_{j+}^{N}\left(t_{k}\right) ; x_{i}^{N}\left(t_{k-1}\right)\right)-\tilde{F}\left(x_{j-}^{N}\left(t_{k}\right) ; x_{i}^{N}\left(t_{k-1}\right)\right)
$$

where the $x_{j+}^{N}\left(t_{k}\right)$ and $x_{j-}^{N}\left(t_{k}\right)$ are defined as previously.

Notice that since the error bound of the filter estimate in 4.10) involves the marginal quantization error: $\left\|\bar{X}_{t_{k}}-\widehat{X}_{t_{k}}^{N}\right\|_{2}$, one must deduce this error from the above construction. We know that the sequence of non-Voronoi quantization $\left(\tilde{X}^{x^{N}}\right)_{N \geq 1}$ defined for every $N \geq 1$ by

$$
\tilde{X}_{t}^{x^{N}}=\sum_{m=1}^{d_{N}} x_{m}^{N}(t) \mathbf{1}_{\left\{W \in C_{m}\left(\alpha^{N}\right)\right\}}
$$

is rate-optimal in $L_{L_{T}^{2}}^{p}(\Omega, \mathbb{P})$ for $p \in[1,2):\left\|\left|X-\tilde{X}^{x^{N}}\right|_{L_{T}^{2}}\right\|_{p}=O\left((\log N)^{-1 / 2}\right)$ (see [15]). One theoretical challenge will be to compute the convergence rate for the marginal functional quantization error.

\section{Numerical illustration}

We deal with numerical experiments by considering an Up-and-out call option in the Black-Scholes model and a local volatility model already considered in [13] and called pseudo CEV model. Recall that in the Black-Scholes framework the stock price process $\left(X_{t}\right)$ is modeled by the following SDE (under the risk neutral probability $\tilde{\mathbb{P}}$ )

$$
d X_{t}=r X_{t} d t+\sigma X_{t} d W_{t}, \quad X_{0}=x_{0}
$$


where $r$ is the interest rate, $\sigma$ the volatility and $W$ a brownian motion under $\tilde{\mathbb{P}}$. For the pseudo CEV model, the dynamics of the stock price process is ruled by the following SDE (under the risk neutral probability)

$$
d X_{t}=r X_{t} d t+\vartheta X_{t}^{\delta} \frac{X_{t}}{\sqrt{1+X_{t}^{2}}} d W_{t}, \quad X_{0}=x_{0}
$$

for some $\delta \in(0,1)$ and $\vartheta \in(0, \underline{\vartheta}], \underline{\vartheta}>0$. The parameter $r$ still be the interest rate and $\sigma(x):=$ $\vartheta \frac{x^{\delta}}{\sqrt{1+x^{2}}}$ corresponds to the local volatility function. We notice that for a fixed $\delta \in(0,1)$, if the initial value of the stock process $X_{0}$ is large enough then the pseudo CEV model is very close to the CEV model

$$
d X_{t}^{\prime}=X_{t}^{\prime}\left(r d t+\vartheta\left(X_{t}^{\prime}\right)^{\delta-1} d W_{t}\right) .
$$

In particular, for numerical tests we will consider that $\vartheta \approx \sigma X_{0}^{1-\delta}$ where $\sigma$ denotes the regular volatility. The only "aim of the really" rough calibration is just to deal with reasonable values to obtain prices close to those given by the Black-Scholes model. For all the experiments we set the interest rate $r$ equal to 0.15 . The maturity is set to $T=1$, the initial value of the stock process $x_{0}=100$ and $\delta=0.5$ (in the local volatility model). For numerics, the solution of the ODE given in (5.8) is approximated by a sixth order Runge-Kutta scheme and marginal quantizations are of size $d_{N}=966$ (corresponding to the optimal decomposition $N_{1}=23, N_{2}=7, N_{3}=3, N_{4}=2$, for the problem (5.6), see [20]).

In the Black-Scholes model we compare the prices computed from the quantization of the continuous Euler process using (5.8) and (5.9) (which prices are referred by QEP prices) with the regular Brownian Bridge method (RBB prices), given the true prices obtained from a semi-closed formula available in [6]. The regular Brownian Bridge (RBB) method is some efficient method to compute expressions like

$$
\mathbb{E} f\left(\bar{X}_{T}, \sup _{t \in[0, T]} \bar{X}_{t}\right) \quad \text { or } \mathbb{E} f\left(\bar{X}_{T}, \inf _{t \in[0, T]} \bar{X}_{t}\right),
$$

based on Proposition 3.1 and consisting (for example for the estimation of $\mathbb{E} f\left(\bar{X}_{T}, \sup _{t \in[0, T]} \bar{X}_{t}\right)$ ) in the following steps :

Set $S^{f}=0$.

for $m=1$ to $M$

- Simulate a path of the discrete time Euler scheme $\left(\bar{X}^{(m)}\right)$ and set $x_{k}=\bar{X}_{t_{k}}^{(m)}, k=0, \ldots, n$.

- Simulate $\Gamma^{(m)}:=\max _{0 \leq k \leq n}\left(G_{x_{k}, x_{k+1}}\right)^{-1}\left(U_{k}^{(m)}\right)$, where $\left(U_{k}^{(m)}\right)_{1 \leq k \leq n}$ are iid with $\mathcal{U}([0,1])$-distribution.

- compute $f\left(\bar{X}_{T}^{(m)}, \Gamma^{(m)}\right)$.

- Compute $S_{m}^{f}:=f\left(\bar{X}_{T}^{(m)}, \Gamma^{(m)}\right)+S_{m-1}^{f}$.

end. $(m)$

with

$$
\left(G_{x, y}\right)^{-1}(1-u)=\frac{1}{2}\left(x+y+\sqrt{(x-y)^{2}-2 T \sigma^{2}(x) \log (u) / n}\right), u \in(0,1) .
$$

Then for large enough $M$,

$$
\mathbb{E} f\left(\bar{X}_{T}, \sup _{t \in[0, T]} \bar{X}_{t}\right) \approx \frac{S_{M}^{f}}{M} .
$$


The numerical results are depicted in Table 2 and Table 3 for varying values of the barrier $L$ and the volatility $\sigma$. The number $M$ of Monte Carlo simulations is set to $10^{6}$. For the quantization methods, the computation times (QEP c.t.) varies from 0 to 3 seconds when $n=10$ and, from 1 to 6 seconds when $n=20$, increasing with the barrier as pointed out in Remark 4.7 However, for the RBB, the computation time is of 2 seconds when $n=10$ and of 5 seconds when $n=20$. The obtained results show that the quantization method may sometimes be competitive with respect to the regular Brownian Bridge method, specially for small number of time discretization steps $n$.

\begin{tabular}{llllll}
\multicolumn{5}{c}{$\sigma=\mathbf{0 . 0 7}, \mathbf{n}=\mathbf{1 0}$} \\
\hline L & True prices & RBB prices & RBB var. & QEP prices & QEP c. t. \\
\hline 105 & $\mathbf{0 . 0 3 4}$ & 0.035 & 0.086 & 0.035 & $<1 \mathrm{~s}$ \\
110 & $\mathbf{0 0 . 5 9}$ & 00.60 & 2.942 & 00.59 & $1 \mathrm{~s}$ \\
115 & $\mathbf{0 2 . 5 8}$ & 02.62 & 15.80 & 02.59 & $2 \mathrm{~s}$ \\
120 & $\mathbf{0 6 . 0 1}$ & 06.11 & 33.54 & 06.03 & $2 \mathrm{~s}$ \\
125 & $\mathbf{0 9 . 5 8}$ & 09.89 & 41.76 & 09.60 & $2 \mathrm{~s}$ \\
130 & $\mathbf{1 2 . 0 7}$ & 12.13 & 43.09 & 12.08 & $3 \mathrm{~s}$ \\
\hline
\end{tabular}

Table 1: Up-and-out Call prices from the quantization method (QEP prices) and the regular brownian bridge method (RBB prices) in the Black-Scholes model for $r=0.15, \sigma=0.07, d_{N}=966, T=1, K=100$, $X_{0}=100, \mathbf{n}=\mathbf{1 0}$ and for varying values of the barrier $L$. QEP c.t. is the quantization method computation time.

\begin{tabular}{llllll}
\multicolumn{5}{c}{$\sigma=\mathbf{0 . 0 7}, \mathbf{n}=\mathbf{2 0}$} \\
\hline L & True prices & RBB prices & RBB var. & QEP prices & QEP c. t. \\
\hline 105 & $\mathbf{0 . 0 3 4}$ & 0.035 & 0.086 & 0.034 & $1 \mathrm{~s}$ \\
110 & $\mathbf{0 0 . 5 9}$ & 00.60 & 2.942 & 00.59 & $3 \mathrm{~s}$ \\
115 & $\mathbf{0 2 . 5 8}$ & 02.59 & 15.80 & 02.59 & $4 \mathrm{~s}$ \\
120 & $\mathbf{0 6 . 0 1}$ & 06.05 & 33.54 & 06.02 & $4 \mathrm{~s}$ \\
125 & $\mathbf{0 9 . 5 8}$ & 09.64 & 41.76 & 09.59 & $5 \mathrm{~s}$ \\
130 & $\mathbf{1 2 . 0 7}$ & 12.10 & 43.09 & 12.08 & $6 \mathrm{~s}$ \\
\hline
\end{tabular}

Table 2: Up-and-out Call prices from the quantization method (QEP prices) and the regular brownian bridge method (RBB prices) in the Black-Scholes model for $r=0.15, \sigma=0.07, d_{N}=966, T=1, K=100$, $X_{0}=100, \mathbf{n}=\mathbf{2 0}$ and for varying values of the barrier $L$.

For the local volatility model we compare the QEP prices with the prices obtained from regular Brownian bridge method. Numerical results are depicted in Tables 4 and 5 for different volatilities and for different values of the barrier. Our reference prices are computed from the regular Brownian bridge method with $10^{7}$ Monte Carlo simulations and 100 times discretization steps. We remark that the quantization method is competitive (because it is faster with the same precision) with respect to the regular Brownian bridge method when the barrier is closed to $X_{0}$. But, the QEP prices become less precise when the volatility and the barrier increase (for example when $\sigma=1.0$ and $L=130$, where the absolute error is of $2 \%$ with respect to RBB method). This might be due to the additional error coming from the estimation of the transition probabilities by formula (5.12) since the conditional law is not known.

Notice that, since we have used sixth order Runge-Kutta scheme to approximate solutions of the 


\begin{tabular}{lllll}
\multicolumn{5}{c}{$\sigma=\mathbf{0 . 1}, \mathbf{n}=\mathbf{2 0}$} \\
\hline L & True prices & RBB prices & RBB var. & QEP prices \\
\hline 105 & $\mathbf{0 . 0 2 9}$ & 0.029 & 0.067 & 0.029 \\
110 & $\mathbf{0 0 . 4 2}$ & 00.43 & 1.933 & 00.42 \\
115 & $\mathbf{0 1 . 7 0}$ & 01.72 & 10.46 & 01.71 \\
120 & $\mathbf{0 3 . 9 5}$ & 03.98 & 26.42 & 03.97 \\
125 & $\mathbf{0 6 . 7 0}$ & 06.76 & 43.82 & 06.72 \\
130 & $\mathbf{0 9 . 3 1}$ & 09.38 & 57.19 & 09.34 \\
\hline
\end{tabular}

Table 3: Up-and-out Call prices from the quantization method (QEP prices) and the regular brownian bridge method (RBB prices) in the Black-Scholes model for $r=0.15, \sigma=0.1, d_{N}=966, T=1, K=100$, $X_{0}=100, \mathbf{n}=\mathbf{2 0}$, and for varying values of the barrier $L$.

$\vartheta=0.7, \mathbf{n}=\mathbf{2 0}$

\begin{tabular}{llllll}
\hline L & Ref. Price & RBB price & RBB var. & QEP price & QEP c. t. \\
\hline 105 & $\mathbf{0 . 0 3 4}$ & 0.034 & 0.085 & 0.034 & $2 \mathrm{~s}$ \\
106 & $\mathbf{0 . 0 7 4}$ & 0.074 & 0.222 & 0.074 & $2 \mathrm{~s}$ \\
107 & $\mathbf{0 0 . 1 4}$ & 00.14 & 0.496 & 00.14 & $2 \mathrm{~s}$ \\
110 & $\mathbf{0 0 . 5 9}$ & 00.59 & 2.949 & 00.59 & $3 \mathrm{~s}$ \\
111 & $\mathbf{0 0 . 8 6}$ & 00.86 & 4.636 & 00.86 & $4 \mathrm{~s}$ \\
112 & $\mathbf{0 1 . 2 0}$ & 01.20 & 6.841 & 01.20 & $4 \mathrm{~s}$ \\
115 & $\mathbf{0 2 . 6 4}$ & 02.66 & 16.34 & 02.66 & $5 \mathrm{~s}$ \\
120 & $\mathbf{0 6 . 2 5}$ & 06.29 & 34.47 & 06.30 & $6 \mathrm{~s}$ \\
125 & $\mathbf{0 9 . 9 8}$ & 10.00 & 41.73 & 10.02 & $7 \mathrm{~s}$ \\
130 & $\mathbf{1 2 . 4 4}$ & 12.45 & 41.65 & 12.46 & $8 \mathrm{~s}$ \\
\hline
\end{tabular}

Table 4: Up-and-out Call prices from RBB and QEP methods in the local volatility model. Model parameters: $r=0.15, \delta=0.5, \vartheta=0.07, d_{N}=966, T=1, K=100, X_{0}=100$ and for varying values of the barrier $L$.

\begin{tabular}{lllll}
\multicolumn{5}{c}{$\vartheta=\mathbf{1 . 0}, \mathbf{n}=\mathbf{2 0}$} \\
\hline L & Ref. Price & RBB price & RBB var. & QEP price \\
\hline 105 & $\mathbf{0 . 0 2 9}$ & 0.029 & 0.067 & 0.029 \\
106 & $\mathbf{0 0 . 0 6}$ & 00.06 & 0.165 & 00.06 \\
107 & $\mathbf{0 0 . 1 1}$ & 00.11 & 0.165 & 00.11 \\
110 & $\mathbf{0 0 . 4 3}$ & 00.43 & 1.966 & 00.43 \\
111 & $\mathbf{0 0 . 6 1}$ & 00.61 & 3.022 & 00.61 \\
112 & $\mathbf{0 0 . 8 3}$ & 00.84 & 4.423 & 00.84 \\
115 & $\mathbf{0 1 . 7 7}$ & 01.77 & 10.89 & 01.78 \\
120 & $\mathbf{0 4 . 1 6}$ & 04.20 & 27.81 & 04.20 \\
125 & $\mathbf{0 7 . 1 1}$ & 07.14 & 45.41 & 07.17 \\
130 & $\mathbf{0 9 . 8 7}$ & 09.90 & 58.17 & 09.92 \\
\hline
\end{tabular}

Table 5: Up-and-out Call prices from RBB and QEP methods in the local volatility model for $r=0.15$, $\delta=0.5, \vartheta=0.1, d_{N}=966, T=1, K=100, X_{0}=100$ and for varying values of the barrier $L$. 
ODE [5.8), there is no hope to improve of price approximations for quantization method by increasing the number $n$ of discretization steps, so that the RBB method will become more competitive when increasing $n$. To improve the estimations for quantization method we must increase the size of $d_{N}$. But, this will increase the computation time and the method will become less faster than the RBB one.

\section{References}

[1] Baldi, P. (1995). Exact asymptotics for the probability of exit from a domain and applications to simulations. The Annals of Applied Probability, 23(4), pp. 1644-1670.

[2] Bally V. \& Pagès G. (2003). A quantization algorithm for solving discrete time multidimensional optimal stopping problems, Bernoulli, 9(6), 1003-1049.

[3] Bally V., Pagès G. \& Printems J., A quantization tree method for pricing and hedging multidimensional American options, Mathematical Finance, 15(1), 119-168.

[4] Bucklew J. A. \& Wise G. L. (1982). Multidimensional asymptotic quantization theory with $r$-th power distribution measures, IEEE Trans. Inform. Theory 28, 239-247.

[5] Callegaro G. \& Sagna A. (2009). An application to credit risk of a hybrid Monte Carlo-Optimal quantization method, preprint.

[6] Conze A. \& Viswanathan. (1991). Path Dependent Options: The Case of Llookback Options, The Journal of Finance, 46(5), 1893-1907.

[7] Corsi M., Pham H. \& Runggaldier W., Numerical approximation by quantization of control problems in finance under partial observations, to appear in Mathematical modeling and numerical methods in finance, special volume of Handbook of Numerical analysis.

[8] Dereich S., Fehringer F., Matoussi A. \& Scheutzow M. (2003). On the link between small ball probabilities and the quantization problem for Gaussian measures on Banach spaces, Journal of Theoretical Probability, 16, 249-265.

[9] Glasserman P., Monte Carlo Methods in Financial Engineering, Springer-Verlag, New York, $596 \mathrm{p}$.

[10] Gobet E. (2000). Weak approximation of killed diffusion using Euler schemes, Stochastic Processes and their Applications, 87,167-197.

[11] Graf S. \& Luschgy H. (2000). Foundations of Quantization for Probability Distributions, Lecture Notes in Mathematics, Vol 1730, Berlin.

[12] Karatzas I. \& Shreve S. E. (1991). Brownian Motion and Stochastic Calculus, Springer-Verlag, New York.

[13] Lemaire V. \& Pagès G. (2008). Unconstrained Recursive Importance Sampling, preprint LPMA 1230.

[14] Luschgy H. \& Pagès G. (2002). Functional quantization of Gaussian processes, Journal of Functional Analysis, 196, 486-531. 
[15] Luschgy H. \& Pagès G. (2006). Functional quantization of a class of Brownian diffusions: a constructive approach, Stoch. Proc. and their Appl., 116, 310-336.

[16] Øksendal B. (1995). Stochastic Differential Equation, 4-th ed., Springer-Verlag, New York.

[17] Pagès G. (1998). Space vector quantization method for numerical integration, Journal of Computational and Applied Mathematics, 89, 1-38.

[18] Pagès G. \& Pham H. (2005) Optimal quantization methods for nonlinear filtering with discretetime observations, Bernoulli,11(5), 893-932.

[19] Pagès G., Pham H. \& Printems J. (2004) Optimal quantization methods and applications to numerical problems in finance, Handbook on Numerical Methods in Finance (S. Rachev, ed.), Birkhauser, Boston.

[20] Pagès G. \& Printems J. (2005). Functional quantization for numerics with an application to option pricing, Monte Carlo Methods and Applications, 11 (4), 407-446.

[21] Pham H., Runggaldier W. \& Sellami A. (2005). Approximation by quantization of the filter process and applications to optimal stopping problems under partial observation, Monte Carlo Methods and Applications, 11, 57-82.

[22] Sagna, A. (2008). Optimal quantization methods with applications to finance. Ph. D. dissertation. http://tel.archives-ouvertes.fr/docs/00/34/20/33/PDF/these.pdf

[23] Zador P. (1982). Asymptotic quantization error of continuous signals and the quantization dimension, IEEE Trans. Inform. Theory, 28, 139-149. 